\title{
90.10. MedBed and its Effect on Cultivated Intestinal Epithelial Cells and Functional Neutrophils
}

\author{
Peter C. Dartsch
}

Dartsch Scientific GmbH, Institute for Cell Biological Test Systems, Auf der Vosshardt 25, D-49419 Wagenfeld, Germany

*Corresponding author: Peter C. Dartsch, Dartsch Scientific GmbH, Institute for Cell Biological Test Systems, Auf der Vosshardt 25, D-49419 Wagenfeld, Germany, E-mail: pc.dartsch@dartsch-scientific.com

\section{ABSTRACT}

Background: Quantum entanglement is a phenomenon in theoretical physics that happens when pairs or groups of particles are generated in such a way that the quantum state of each particle cannot be described independently of the others even when the particles are separated by a large distance.

Material and methods: The 90.10. MedBed is a virtual bed that is quantum entangled with a 90.10.-CUBE by a quantum processor, the $9010 \mathrm{MedBedOS}$. The 90.10.-CUBE was located in Akumal Quintana Roo, Mexico, 8,603 kilometers air-line distance from the cell culture laboratory conducting the tests. The 90.10. MedBed is a virtual bed that is quantum entangled with a 90.10.-CUBE by a quantum processor, the $9010 \mathrm{MedBedOS}$. By the process of 90.10 . quantum entanglement, the 90.10. CUBE's basic potential is transferred to the 90.10. MedBed. For the present in vitro-study with intestinal epithelial cells and functional neutrophils - at laboratory conditions instead of a bed - seeded cells in the culture dishes were quantum entangled with the 90.10. MedBed, i.e. the system was installed on the dishes and activated in the laboratory to conduct the study. Control dishes were not treated and were incubated in the same incubator with a distance of at least 30 to $40 \mathrm{~cm}$ to the treated dishes.

Results: For intestinal epithelial cells, a better regeneration process was determined demonstrating that 90.10 . MedBed was able to promote healing processes of the intestinal cell layer and, thus, to promote intestinal and systemic health and wellbeing. The second experimental series with functional neutrophils as inflammationmediating cells demonstrated a marked decrease in the generation of reactive superoxide anion radicals by exposure to 90.10. MedBed quantum entanglement. In vivo, this influence might have a positive impact on the healing process of complicated secondary wounds or on (chronic) inflammatory processes directly.

Conclusions: The results clearly demonstrate the effectiveness of the 90.10. MedBed which was quantum entangled with a 90.10 .-CUBE by a quantum processor, the 9010MedBedOS, on the cellular level. The results confirm and complement previous findings on the effect of 90.10. quantum entanglement on cultured organ-specific cells in which we demonstrated an increased regeneration of cultured connective tissue fibroblasts.

\section{Keywords}

Quantum energy

90.10. MedBed

Quantum entanglement Intestinal epithelial cells

IPEC-J2

Cell regeneration

Functional neutrophils

HL-60

Oxidative burst Cell culture 


\section{INTRODUCTION}

As stated by Horodecki et al. [1], ,it was Einstein, Podolsky, and Rosen (EPR) and Schrödinger who first recognized a special ... feature of quantum machinery which lies at the center of interest of physics of the 21 st century“ "[2,3]. It describes a physical phenomenon that occurs when a group of particles interact in such a way that the quantum state of each particle of this group cannot be described independently from the state of the others. This also includes a state when the particles are separated by a long distance. The term for this phenomenon is now called ,quantum entanglement" and was originally called by Schrödinger et al. [4-6] as „Verschränkung“. Recent advances in quantum information theory reveal the deep connections between entanglement and thermodynamics, many-body theory, quantum computing and its link to macroscopicity [7].

The 90.10. MedBed is a virtual bed that is quantum entangled with a 90.10.-CUBE by a quantum processor, the 9010MedBedOS. The 90.10.-CUBE consists of a holder with two levels and two times six aluminum modules. The modules have been treated in a special quantum physical process so that they radiate quantum energy and create an energy field of a certain strength in the space between the upper and lower levels. In this space, any matter can be quantum-physically treated and thus optimized in its structure and properties. Quantum-physical refinement can also be carried out over a long distance. This is achieved by using the possibilities of quantum entanglement. Quantum energy and frequencies are then teleported into matter. By the process of 90.10. quantum entanglement, the 90.10 . CUBE's basic potential is transferred to the 90.10 . MedBed.

For the present in vitro-study with intestinal epithelial cells and functional neutrophils at laboratory conditions instead of a bed, seeded cells in the culture dishes were quantum entangled with the 90.10. MedBed, i.e. the system was installed on the dishes and activated in the laboratory to conduct the study.

\section{MATERIAL AND METHODS}

\section{Quantum entanglement by 90.10. MedBed}

The quantum processor 9010MedBedOS was located in Akumal Quintana Roo, Mexico, 8,603 kilometers air-line distance from the cell culture laboratory conducting the tests. For the quantum entanglement, photographs of the cell culture dishes with the seeded cells were used. Control dishes were not exposed, but were also incubated in the same incubator with a distance of at least 30 to $40 \mathrm{~cm}$ to the 90.10 . MedBed quantum entangled dishes.

\section{Intestinal epithelial cells}

The investigations were conducted with IPEC-J2 cells (ACC-701; Leibniz Institut, DSMZ, Braunschweig, Germany). The cells were routinely grown in Dulbecco's Modification of Eagles Medium supplemented with $10 \%$ growth mixture and $0.5 \%$ gentamycin and cultivated in an incubator at $37^{\circ} \mathrm{C}$ in an atmosphere of $5 \% \mathrm{CO}_{2}$ and $95 \%$ air at nearly $100 \%$ humidity. The cells were routinely cultivated as mass cultures and were regularly subcultured twice a week with fresh culture medium. For the experiments, cells were taken from $80-90 \%$ confluent mass cultures.

The granulation phase, characterized by the occurrence of migration and proliferation of epithelial cells for closing a defect $[8,9]$, was simulated. For this purpose, cells were seeded at a density of 100,000 cells $/ \mathrm{ml}$ into the three individual compartments of a silicone 3 well-culture insert made (ibidi, Gräfelfing, Germany). The single compartments of the inserts are separated by a $500 \mu \mathrm{m}$ thick silicone bar with an outer silicone frame of $700 \mu \mathrm{m}$. Due to the special adhesion area, an insert adheres firmly to the bottom of a culture dish and forms a distinct cell-free area (artificial wound), which the cells can colonize by migration and proliferation.

Upon reaching confluency within 48 hours after cell seeding, the silicon frames were removed with tweezers to achieve a sharp edge of the cell-free area between the compartments. The intestinal epithelial cells in the dishes with and without 90.10. MedBed quantum entanglement were allowed to migrate and proliferate for 7 hours. Finally, cell cultures were fixed with $100 \%$ methanol, stained with Giemsa's azur eosin methylene blue solution (Merck, Darmstadt, Germany) and air-dried. The colonized area was examined by micrographs and calculated by a specialized software with artificial intelligence from KML Vision, Graz, Austria (IKOSA AI software). Eight independent experimental series $(n=8)$ during an experimental period of three weeks were conducted.

\section{Functional neutrophils}

The investigations were conducted with human promyelocytes (cell line HL-60; ACC-3; ECACC 98070106; Leibniz Institute DSMZ, Braunschweig, Germany). The cells were routinely cultivated in RPMI 1640 culture medium with 10\% growth mixture and $0.5 \%$ gentamycin and incubated in an incubator at $37^{\circ} \mathrm{C}$ and an atmosphere of $5 \% \mathrm{CO}_{2}$ and $95 \%$ air and almost $100 \%$ humidity. The non-adherent cells were routinely cultivated as suspended mass cultures and were subcultured twice a week. The cells were differentiated into inflammationmediating cells (= functional neutrophils) able to generate superoxide anion radicals in the course of an oxidative burst by treatment with $1.5 \%$ dimethyl sulfoxide for six days. 
For the last 24 hours of differentiation, the flasks with the cells were exposed to 90.10. MedBed quantum entanglement. Cells without quantum entanglement served as corresponding controls. Finally, cells were collected and washed by subsequent centrifugation steps at $190 \mathrm{x}$ g. By adding a phorbol ester (phorbol-12-myristate-13-acetate, SigmaAldrich, Taufkirchen, Germany) to a specific reaction mixture,

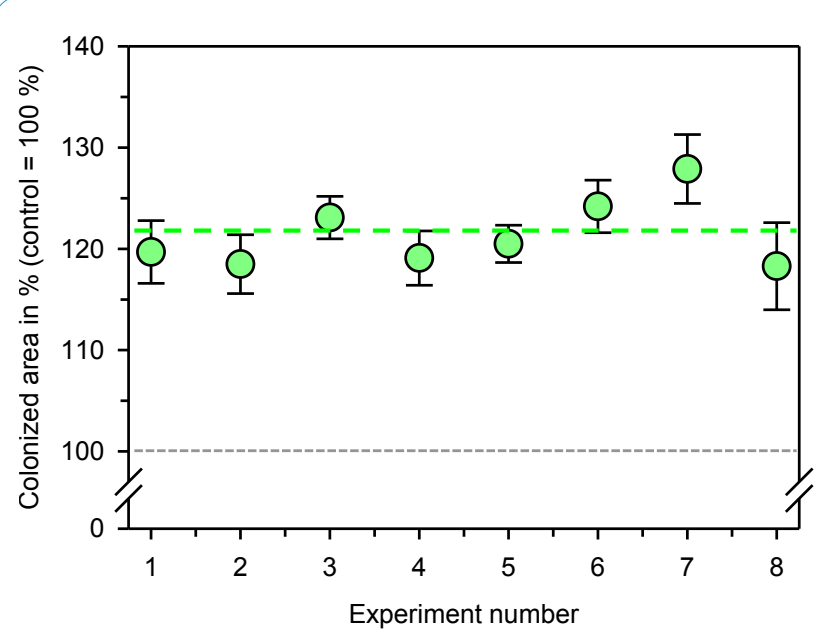

Figure 1: Graphical presentation of the relative increase of colonized area in comparison to untreated controls in 8 independent experiments by use of 90.10. MedBed quantum entanglement during the 7 hour period of regeneration. The untreated control is set as $100 \%$ (dashed grey line). The mean value of all 8 experiments is marked by the dashed line in green. Data points represent mean value \pm standard deviation of each experiment. the differentiated cells were stimulated to generate superoxide anion radicals by an oxidative burst.

The radicals cause a cleavage of a tetrazolium dye (WST-1; Sigma-Aldrich, Taufkirchen, Germany) that was also added to the reaction mixture. The cleavage of the dye is directly related to the amount of oxygen radicals, i.e. the more reactive radicals are present, the greater the cleavage of the dye and the change in optical density (=colour). If the radicals generated by the cells are inactivated by the exposure to 90.10 . MedBed quantum entanglement, the optical density changes less strongly.

The optical density was recorded at $\mathrm{t}=0$ and definite time points with an Elisareader (BioTek ELx808 with software Gen 5 version 3.00) and calculated with Microsoft Excel 2016.

\section{STATISTICAL ANALYSIS}

Statistical analysis was done using the parameter-free twotailed Wilcoxon-Mann-Whitney rank sum test.

\section{RESULTS AND DISCUSSION}

\section{Regeneration of intestinal epithelial cells}

The colonized area was significantly increased by 90.10 . MedBed quantum entanglement in comparison to the untreated controls (Figure 1). The increase of all 8 experiments was 21.4 $\pm 3.4 \%$ (mean value \pm standard deviation; $p \leq 0.01$ ). At first sight, this does not seem to be a respectable increase. But it must be considered that this is a two-dimensional evaluation which is much more strengthened when taking the migration distance of the cell layers into account which is more impressively represented by the micrographs in Figure 2.
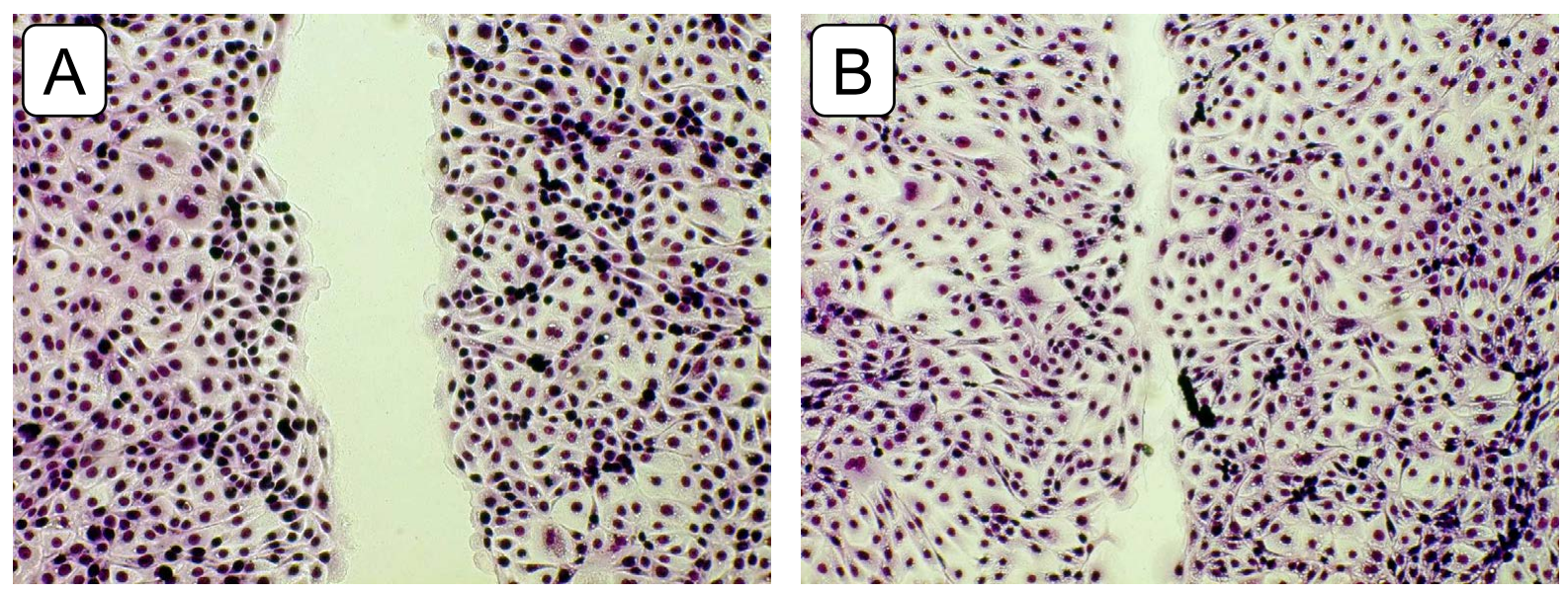

Figure 2: Micrographs of the regeneration/wound healing process after fixation and staining of intestinal epithelial cells after 7 hours of regeneration. (A) Colonized area of the untreated control. (B) Colonized area of a representative cell culture after exposure to 90.10. MedBed quantum entanglement. Note the markedly increased closure of the cell-free area after 90.10. MedBed quantum entanglement. Olympus IX 50 inverted microscope with Planachromate 10x and Olympus E-10 digital camera at 4 megapixel resolution and bright field illumination. 


\section{Research Article}

The intestinal epithelium, which is only one cell layer thick, has two essential tasks. The first is to create a physical barrier between the contents of the intestinal lumen and the rest of our body. The second is to ensure an efficient absorption of essential nutrients from the gut lumen and to produce mucus, anti-microbial peptides and cytokines with both protective and immune-regulatory properties. Thus, a reduced barrier function may have far reaching consequences, not only for intestinal, but also for systemic health [10].

Prompted by this background cultured intestinal cells were used to examine the effect of 90.10. MedBed on the regenerative potential of the epithelal barrier. The IPEC-J2 cell line was chosen, because "the IPEC-J2 cell line is unique as it is derived from the small intestine and is neither transformed nor tumorigenic in nature. IPEC-J2 cells mimic the human physiology more closely than any other cell line of non-human origin" [11]. The cells were originally isolated in 1989 by Helen Berschneider at the University of North Carolina [12]. The advantage of the IPEC-J2 cell line as an in vitro model originates from its morphological and functional similarities with intestinal epithelial cells in vivo [13]. The epithelial cells of the intestinal barrier have a high turnover rate, because they are quite sensitive against alterations of their endogenous environmental conditions involving a deficiency of the epithelium and immune/inflammation mediating cells [14].

As observed in the present study, 90.10. MedBed quantum entanglement resulted in an increased regeneration of intestinal epithelial cells which might also increase the turnover rate of the cells in vivo. Consequently, the use of the 90.10. MedBed might result in an improved barrier function and intestinal health, which in turn, improves well-being and systemic health. The results confirm and complement previous findings on the effect of 90.10. quantum entanglement on cultured organ-specific cells in which we demonstrated an increased regeneration of cultured connective tissue fibroblasts [15].

\section{Oxidative burst of inflammation-mediating cells}

As depicted in Figure 3, exposure of the inflammationmediating cells to 90.10. MedBed quantum entanglement significantly reduced the generation of superoxide anion radicals by an oxidative burst from $100 \%$ (=untreated control) to $58.2 \pm 7.4 \%$ (mean value \pm standard deviation; $\mathrm{p} \leq 0.01$ ).

Neutrophil granulocytes (polymorphonuclear neutrophils, PMN) represent the largest group of leukocytes. They build the first line of defense against pathogenic microorganisms, fighting them by phagocytosis via release of antimicrobial molecules and generate reactive oxygen species by an oxidative burst [15]. Attracted by chemical substances such

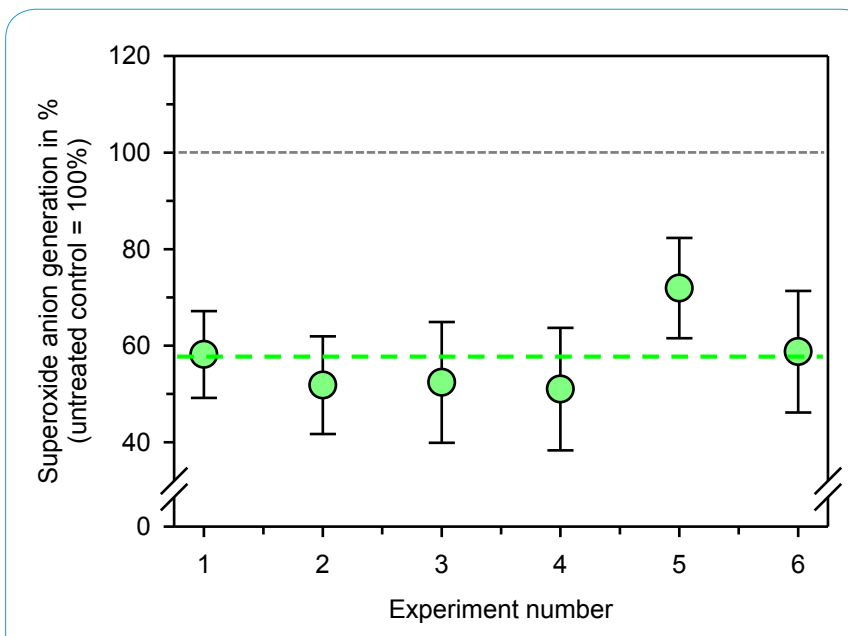

Figure 3: Graphical presentation of the generation of superoxide anion radicals by use of 90.10. MedBed quantum entanglement in comparison to untreated controls in 6 independent experiments. The superoxide anion generation of the untreated controls is set as $100 \%$ (dashed grey line). The mean value of all 6 experiments is marked by the dashed line in green. Data points represent mean value \pm standard deviation of each experiment.

as specific chemokines or cytokines which occur during the inflammatory process, these cells can migrate from the blood into the inflamed tissue and generate superoxide anion radicals in the tissue $[16,17]$. The radicals cause further tissue destruction (necrosis) in the inflamed tissue and might cause a progression of the inflammatory process with decelerated wound healing. For an overview of the role of neutrophils in health and disease, see [18-20].

We used an in vitro model which represents one facet of the inflammatory process to investigate whether 90.10. MedBed quantum entanglement was able to reduce endogenous superoxide anion radical generation better than untreated control cells. The reduced radical generation of functional neutrophils is comparable to an anti-inflammatory effect in the tissue. However, the effect is within a range which should not markedly affect the innate immune system of the blood as a first defense against microbial pathogens.

\section{CONCLUSIONS}

The investigations of the present study demonstrate very impressively that 90.10. MedBed quantum entanglement causes significant cellular effects over an airline-distance of 8,603 kilometers. For intestinal epithelial cells, a better regeneration process was determined demonstrating that 90.10. MedBed quantum entanglement is able to promote healing processes of the intestinal cell layer and, thus, to improve intestinal and 
systemic health and well-being. The second set of experiments with inflammation-mediating cells demonstrated a marked decrease in the generation of reactive superoxide anion radicals by exposure to 90.10 . MedBed quantum entanglement. In vivo, this influence might have a positive impact on the healing process of complicated secondary wounds or on (chronic) inflammatory processes directly.

\section{REFERENCES}

1. Horodecki R, Horodecki P, Horodecki M, Horodecki K (2009) Quantum entanglement. Rev Mod Phys 81: 865-942.

2. von Neumann J (1932) Mathematische Grundlagen der Quantenmechanik. Springer, Berlin.

3. Einstein A, Podolsky B, Rosen N (1935) Can quantum-mechanical description of physical reality be considered complete?. Phys Rev 47: 777-780.

4. Schrödinger E (1935) Die gegenwärtige Situation in der Quantenmechanik. Naturwiss 23: 807.

5. Schrödinger E, Born M (1935) Discussion of probability relations between separated systems. Math Proc Camb 31: 555-563.

6. Schrödinger E, Dirac PAM (1936) Probability relations between separated systems. Math Proc Camb 32: 446-452.

7. Vedral V (2014) Quantum entanglement. Nature Physics 10: 256259.

8. Witte M, Barbul A (1997) General principles of wound healing. Surg Clin North Am 77: 509-528.

9. Wallace HA, Basehore BM, Zito PM (2019) Wound Healing Phases. In: StatPearls. StatPearls Publishing, Treasure Island (FL).
10. Lea T (2015) Epithelial cell models; general introduction. In: Verhoeckx K. et al. (eds) The Impact of Food Bioactives on Health. Springer, Cham, pp. 95-102.

11. Vergauwen H (2015) The IPEC-J2 cell line. In: Verhoeckx K. et al. (eds) The Impact of Food Bioactives on Health. Springer, Cham, pp. 125-134.

12. Berschneider HM (1989) Development of normal cultured small intestinal epithelial cell lines which transport $\mathrm{Na}$ and $\mathrm{Cl}$. Gastroenterology 96: A41.

13. Schierack P, Nordhoff M, Pollmann M, Weyrauch KD, Amasheh S, et al. (2006) Characterization of a porcine intestinal epithelial cell line for in vitro studies of microbial pathogenesis in swine. Histochem Cell Biol 125: 293-305.

14. Bhattacharyya A, Chattopadhyay R, Mitra S, Crowe SE (2014) Oxidative stress: An essential factor in the pathogenesis of gastrointestinal mucosal diseases. Physiol Rev 94: 329-354.

15. Nathan C (2006) Neutrophils and immunity: Challenges and opportunities. Nat Rev Immunol 6: 173-182.

16. Weiss SJ (1989) Tissue destruction by neutrophils. N Engl J Med 320: 365-376.

17. Mortaz E, Alipoor SD, Adcock IM, Mumby S, Koenderman L (2018) Update on neutrophil function in severe inflammation. Front Immunol 9: 2171.

18. Selders GS, Fetz AE, Radic MZ, Bowlin GL (2017) An overview of the role of neutrophils in innate immunity, inflammation and host-biomaterial integration. Regenerat Bio-mater 4: 55-68.

19. Hellebrekers P, Vrisekoop N, Koenderman L (2018) Neutrophil phenotypes in health and disease. Eur J Clin Invest 23: e12943.

20. Dartsch PC (2021) Effect of 90.10. quantum entanglement on regeneration of cultured connective tissue fibroblasts. Biomed J Sci Tech Res 38: 30841-30844. 